\title{
Training for virtual exchange
}

\section{Christopher Brighton ${ }^{1}$}

\section{Abstract}

7 he article describes the training program introduced at East Carolina University (ECU) for the Global Understanding (GU) program and the Global Partners in

Education (GPE) consortium. The aim of the training was to reinforce instructors' intercultural skills and build on the well-known methodology of virtual exchange. Over one semester, the instructors attended face-to-face training sessions. These two-hour meetings focused on several pre-identified areas which needed increased instructor awareness: Cultural Intelligence (CQ), reflections, and classroom management, as well as teamwork and collaboration. The general outcomes of the training have seen an improvement in program delivery, classroom interaction, and student support. Additionally, the work in ECU highlights the need for intercultural training to be part of virtual exchange preparation alongside the well-known guides which provide instructors with the structure for conducting this type of modality. As is shown in this report, increased instructor awareness leads to more successful virtual exchange program delivery, pastoral support, and learner outcomes.

Keywords: intercultural communication; cultural intelligence; training; GVE methodology; classroom management.

1. Xi'an Jiaotong-Liverpool University, Suzhou, China; christopher.brighton@xjtlu.edu.cn; https://orcid.org/0000-0002-3110-3841

How to cite this article: Brighton, C. (2020). Training for virtual exchange. Journal of Virtual Exchange, 3 (SI-IVEC2019), 69-79. https://oi.org/10.21827/ jve. 3.35810 


\section{Introduction and background}

As the field of Global Virtual Exchange (GVE) has expanded in recent years, many manuals and guides have been produced to help faculty become acquainted with the methodology. The most wellknown are the Collaborative Online International Learning (COIL) guides produced by the SUNY network (Faculty Guide for COIL course development, v.1.42); however, there are others available which either use the COIL as a basis or build their own structure. Most of these guides provide a step-by-step approach to the 'what' of creating a GVE. They refer to considerations which should be paid attention to when building a partnership and incorporating GVE into the classroom structure. The SUNY COIL guide, for example, explains what faculty should consider: issues of time, language, course content, assessment, technology, expectations, and administrative issues. The University of Washington Bothell's online COIL guide adds power imbalances, such as language and academic level, to the considerations (COIL, 2019).

The guides are a good foundation for anyone wanting to start in the field and cover much of the structural considerations necessary in building a GVE course. However, the guides pay little attention to an essential part of the GVE methodology - intercultural awareness and communication, which can be considered the be the 'how' of GVE. The focus of the training guides is often on preparing instructors to ensure successful content exchange and the discussion of material relevant to the course. Although instructors do acknowledge the relevance of intercultural outcomes through working in the GVE modality, the development of intercultural skills is often an additional outcome of the GVE process.

The building of intercultural skills, which is central to the GVE methodology, is often ignored or taken for granted. The often-held assumption is that instructors who would be attracted to being involved in a GVE program would have a global outlook and have abilities to work interculturally. It is supposed that instructors have developed their own intercultural skills through the negotiation and development of working with an overseas partner. However, instructors, who are specialists in their field, often lack knowledge of intercultural methodology, and cannot provide the support that students need when working in this environment.

Students engaging in this modality need support to develop their intercultural skills from the get-go. Most students will have had little prior experience in working with overseas partners, dealing with time zone differences, language issues, and other cultural differences. To assume that the students' intercultural skills will be built through discussions and interaction within the modality fails to understand the

2. http://www.ufic.ufl.edu/UAP/Forms/COIL_guide.pdf 
complexity of becoming an intercultural communicator. Instructors, therefore, need to be trained not only on the what GVE is, but also on how to support students in their intercultural development.

The GU program, run by the GPE consortium at ECU, recognized the need for instructor development in intercultural methodology. From Fall 2018, the author has worked to implement a system-wide badging program, which will deliver online professional development for all instructors (GPE, 2019). The first level of the program is designed to cover the 'what' of GVE methodology with training specific to the GU Core. The second and third levels are focused on the 'how' of GVE: level two contains trainings on the best practices of intercultural skill building; level three is a portfolio approach of material to illustrate an instructor's work in building intercultural skills. These three levels work in unison to create a training structure that will strengthen instructor competences and knowledge, not only of the what, but also the how of working in a GVE environment.

Although the GU program is not built around specific ideas of intercultural theory, much of the literature is relevant to the training. Byram's (1997) concept of savoir discusses the ways in which intercultural actors utilize their 'knowledges', or savoirs, when communicating with others. Byram's (1997) argument stresses the need for transferable knowledge, versus static factual knowledge, as intercultural actions occur with more frequency and across a more diverse global population. His framework provides educators with guidance on the areas for learners to strengthen. Further, Gudykunst and Kim (1992) stress that intercultural communication is a process of unlearning what is already known and working to assimilate new information, focusing on a psychological, rather than a communicative, dimension of intercultural skills. Their work illustrates the need for socio-cultural development and the challenges communicators face in building intercultural competences. Both these two approaches are included in the methodology for GU with a need for all learners to build both communicative and socio-cultural skills.

Additionally, other aspects of intercultural skill theory impact the methodology for GU courses. For example, Ting-Toomey's (1999) concept of identity negotiation is relevant, as students are often involved in an intercultural setting for the first time, and have to manage the specifics of the environment. Further, Kramsch (1993) speaks about the idea of a 'third place' of intercultural communication, where learners build a construct which includes their native culture and their target culture. Discussing bilingual learners in Canada, Kramsch's concept of the third place has been used by methodologists to discuss the role of the instructor in the classroom as a 'cultural mediator' (Bandura, 2007).

The role of cultural mediator is essential, as the GU classroom is a 'third place' construct. Instructors, therefore, act as cultural facilitators in guiding their students through the process of working with 
their partners in the GU program. Discussions are fluid, and students require intercultural skills to engage with their overseas partners and synthesize the information being learned. The instructor acts as a guide to the students, and must take a flexible approach in managing the classroom interaction, as the focus is on student driven discussion rather than instructor led. Therefore, the focal role of the instructor in GU is to help the students process and synthesize the content of the discussions, rather than to steer the conversation in a certain direction. This is where the necessity of training is essential in not only working with instructors on their roles in supporting their students, but also in providing them with insight into their own intercultural skills.

The rationale behind the development of the training stems from evaluating the outcomes of GU and the program's specific requirements. During a semester, one cohort of approximately 16-to20 students works with a similar cohort from an overseas partner for six meetings of 50-to-75 minutes, usually concluded in a three-week period and using English as the primary language of communication. Scheduling permitting, a cohort works with up to three different partners from a diversity of cultures. In synchronous video and online chat groups, students have the freedom to explore multi-disciplinary topics on College Life, Family Life, the Meaning of Life, and Stereotypes with their partners in any manner they see fit. As a student driven program, the structure puts more emphasis on the need for students to build intercultural skills in flexibility, collaboration, and effective communication with the culturally diverse partners to achieve the course goals. Consequently, the program methodology requires instructors to have the pedagogical tools to support students involved in the program to build transferable intercultural skills to maximize learning outcomes.

The GPE recognized that the success of student outcomes was linked to teachers who could not only engage students in the content of the course, but also provide pastoral support in the student's development of intercultural competence. At present, the GU program uses the CQ survey (Dyne, Ang, \& Koh, 2008) to evaluate student learning outcomes across the network. Despite internal evaluation of the pre- and post-course surveys indicating student development in the four dimensions of CQ - knowledge, strategy, action, drive - it was felt that, in certain situations, instructors did not maximize the potential intercultural learning outcomes. Limited analysis indicated that students who showed the highest improvement in their CQ scores had instructors that maximized the intercultural learning outcomes. Furthermore, observation of instructor delivery illustrated a wide range of interpretations of the idea of intercultural communication, with some instructors focused on specific areas of study rather than the development of transferable skills. Instructors often favored the teaching of stereotypical or prejudicial facts of a partner country rather than providing the students with the skills to adapt and become flexible to different situations. 
With all the above rationale considered, in the spring semester of 2019, a pilot training program was developed to raise instructor awareness of intercultural skills. Four areas were identified as key to the successful delivery and strengthening of the overall GU program quality: (1) CQ; (2) reflections; (3) classroom management; and (4) teamwork and collaboration.

The training was supported by a grant provided by Global Academic Initiatives, the unit in ECU which manages and delivers the GPE. All ECU instructors of GU were required to attend the workshops and provide feedback. Although it was known that the modality of the GPE training program was to be delivered online, it was felt that working with ECU instructors face-to-face would allow for greater discussion of the content prior to digitization. Additionally, the aim was for ECU instructors to set an example and illustrate best practices for the network as they work with the vast majority of the GPE partners each semester. The following is a description of the aim and activities undertaken in each of the trainings.

\section{CQ}

The training for CQ enabled the concepts associated with the CQ scale (Dyne et al., 2008) to be discussed, providing the instructors with a basic foundation in key terminology. The scale analyses skills in four areas: drive (motivational), action (behavioral), strategy (metacognitive), and knowledge (cognitive). Overall CQ is "defined as the ability to function effectively in situations characterized by cultural diversity" (Ang \& Dyne, 2008, p. 3). The concepts of CQ relate to wider theories of intercultural skill development, such as Byram's (1997) savoir approach and Gudykunst and Kim's (1992) socio-cultural ideas of learner assimilation.

An important aspect of the CQ model is that it shows the interconnected nature of the intercultural skills. It provides a visual idea of learners having a toolbox of skills from which to draw in different situations. The model emphasizes that culture-specific knowledge is not essential and that, when working interculturally, it is more about building on experiences. The CQ assessment, like other self-assessment frameworks, talks about knowledge in general terms - such as whether the learner knows about religious behavior, legal systems, or shopping culture in other cultures, etc. The focus of CQ, as with all of the many approaches in intercultural communication, is primarily emotional, and relates to how the learner adjusts to the different situations and manages the experiences of working with other cultures. In this manner, the training emphasized the emotional aspect, and focused the instructors toward examining how their students dealt with the complexity of feelings associated with working interculturally. 
Utilizing a board game structure, the participants were asked to place 16 statements in one of the four CQ dimensions. The statements were taken from the CQ self-assessment questionnaire, and there were an equal number for each category. The instructors initially assumed that the majority of statements were in the dimension of knowledge, with very few statements seen to be part of Action, Strategy, or Drive. During discussion, the instructors admitted that the more abstract concepts were more difficult to understand. Most essentially, they unanimously expressed their concerns of evaluating the soft-skill concepts, and discussed the challenge of adding evaluation of more abstract ideas into their course rubrics. Several instructors explained they were less inclined to include evaluation criteria on the soft skills of intercultural communication in their courses because it was a subjective assessment.

However, it was agreed that the soft skills were an essential part of the GU classroom process and needed to be considered for evaluation. Most importantly, the training did lead to instructors understanding the idea of students building a toolbox of intercultural skills to utilize at different times and in different situations. Most importantly, the training raised understanding of the concept of knowledge and the different types of knowledge - cognitive and metacognitive - that are part of CQ. Furthermore, the consensus after discussion was that more work in the classroom was needed to help guide students through CQ dimensions, thus leading to the second training on reflections.

\section{Reflections}

Building from the discussion of CQ, the second part of the training program aimed to help instructors work on their evaluation of student skills. In the GU Core, there is a requirement that all students involved in the GU program complete some form of reflective exercise after each link. The flexibility of options provides instructors with a range of approaches, from logs and journals to vlogs or short surveys. However, from the methodology of the GU program, effective reflection is necessary to assist students in their synthesis of the material and in assimilating the new cultural knowledge. Furthermore, reflections provide learners with an opportunity to consider the different situations they have faced and their strategy for working toward a successful outcome.

Although there is no one correct way of doing reflective work, to be effective toward the goals of building intercultural skills, the student reflections should focus more on the metacognitive aspects, exploring emotions, challenges, and strategies, than being a record of the details of the links. A properly constructed reflective activity would allow the students a space to report on their assimilation of the material, for example, by mentioning interesting or new things they had learned, 
as well as allowing the students a safe forum to express their emotions and thoughts. Ideally, reflections should lead toward some form of group exercise and discussion, where the instructor can provide guidance to overcome concerns and highlight successful work.

Two models of reflection were presented in the training: the Describe, Examine, and Articulate Learning (DEAL) model (Ash \& Clayton, 2009) and the Intercultural Competence Assessment (INCA) portfolio approach (Byram, Kuhlmann, \& Muller-Jacquier, 2009). The DEAL model uses guided questions with the intention to provide a framework for students. With careful design using the DEAL concept, students objectively describe the situation, examine the skills they used in working on the situation, and then explain their overall experience.

The INCA model, developed by Byram et al. (2009), comes complete with an assessment rubric and observational model for the instructor to work alongside the learner in the classroom. This approach requires the use of recorded materials from the videoconferences to help the student decipher their actions and behavior. Furthermore, the combination of teacher observation and student reflection allows the comparison of notes leading to an understand of how they reacted to certain situations. INCA also encourages dialogue through exploration of areas of disagreement between student and instructor, as well as student to student. This leads to a good discussion board or weekly debriefing class.

The training focus was to understand that the aim of the reflection was to allow them to explore their development of intercultural skills. Reflections encourage learner self-awareness. Space is given for students to record their experiences and examine their actions to understand the success or failure of the communication. In a setting where instructors use either classroom or an e-Learning forum discussion, students can be encouraged to share their thoughts and see how colleagues viewed the same situation from a different perspective. With additional instructor-led discussion, the students can build understanding of how a certain scenario developed and what the essential take-a-way items are for the future. Intercultural skills are built through the mindful reflection of behavior in each situation and then the understanding of how that situation can be explored using different responses.

The outcome of the training was to further develop the idea of an intercultural toolbox and the variety of dimensions connected with intercultural skill development. Most importantly, the training emphasized to the instructors their need for flexibility in the classroom. The instructors understood that they should encourage learners to accept that not all the communication will be comfortable and that different factors influence the outcomes. Additionally, reflections reinforced the importance of an ongoing dialogue between students and instructors concerning progress and development. 


\section{Classroom management}

With the idea of giving learners the space to explore their intercultural development, the classroom management of the GU program becomes especially important. The methodology of GU is to create a student-directed learning environment with instructors acting as facilitators rather than teachers. Student voices and opinions are dominant, with instructors not delivering a lecture or moderating discussions to pre-determined answers. For many instructors, this is a difficult transition to take as they 'give-up' traditional aspects of classroom management. Some instructors struggle with the direction that students take the discussion, as well as with the opinions expressed or with silence as students struggle to build rapport. This leads them to become involved, changing the nature of the classroom by directing the conversation toward their own goals. As a result, in some situations, students do not explore their own intercultural skill development, as the instructor manages the situation on their behalf.

To illustrate these issues and present the nuances of teacher intervention, the training on classroom management used several scenarios that are part of the GU program environment. These scenarios concerned four aspects of the GU environment that point to the type of methodology and approach necessary. The first scenario concerned Effective Participation with the situation of a group of students with different communication styles. The second scenario concerned Conversations and a situation where a student ignored their partner's questions to lead the conversation in a different direction. The third scenario focused on a Taboo Topic and dealing with situations that could be culturally sensitive or create a potential conflict, not only with partners, but also among the students in class. The final topic illustrated a challenge instructors face relating to teacher intervention in discussions.

As the situations were based on GU scenarios, the training allowed instructors to examine their own responses and hear from colleagues about different approaches. The variety of techniques and strategies used allowed instructors to understand the importance of student-directed learning. It was widely agreed that silence is not something to be feared in the GU classroom, but to be embraced, as students will work to fill the gap once they are aware that the instructor will not intervene. As student opinions are key, the instructors understood the importance to work toward a nonjudgmental environment. Students should be allowed the freedom to explore, and questions and issues must be delivered in a respectful manner. The training emphasized the point that instructors should allow the students to evaluate the responses of their partners to decide if further discussion of the topic is appropriate.

Appropriate classroom management provides students with the space to develop their intercultural skills in a structured and safe environment. The GU modality emphasizes the importance of students 
building rapport with partners through communication to build their intercultural skills. It is at the student's discretion to explore the topics and direct the conversation as they feel is appropriate. Responding to their partners' verbal and non-verbal cues in videoconferencing is an important part of intercultural skill development. Instructors, although they are often used to being in control of the direction of conversation, have to take on a different role to encourage participation, stepping in when a student demonstrates a lack of intercultural skills in managing a situation, and acting more as a moderator leading conversation in a direction with a clear learning goal.

\section{Teamwork and collaboration}

GU program instructors are expected to lead by example and show students appropriate teamwork and collaborative skills when working on the program. There are two main areas where instructors need to demonstrate their leadership. First, partner instructors are required to work together in confirming the details of their links. Although the classes are centrally scheduled, there is a need for a working rapport to develop and for negotiation in several areas. In this sense, the importance of the teamwork and collaboration between partner instructors is to effectively establish the links and build the safe environment for student collaboration. The second area of leadership is with the technology support staff, who work alongside instructors to ensure that the links can take place. The technology support is important, as it allows the instructor to focus on their classroom management and troubleshoot issues before they become a problem.

Using different visualizations to show the different team relationships, the training highlighted the interaction between the different teams, and furthered the understanding of the dynamics. It emphasized and illustrated the chain of communication between the different teams to the success of the links. Technology support and instructors need to ensure that they have a working relationship with their partners to ensure that problems are dealt with in a timely manner before they become an issue. The training also brought to attention an often-overlooked aspect of working on the GU program. The GU Core outlines the minimum expectations of student participation in the program, to which all partners are required to adhere. Problems arise when individual institutions expect partner students to follow and complete other requirements, rather than following the GU Core.

Additionally, the training demonstrated how communication between partners can lead to confusion. Given that English is the language of communication, the training examined how email messages can be misinterpreted by non-native English speakers. Instructors voiced their surprise at how their email communication style, such as choice of words and use of phrases, can influence their partner. 
This brought to their attention the linguistic aspect of intercultural communication and focused on the need for effective communication between partners to ensure clarity.

\section{Training outcomes}

The training highlighted the need for instructors to have access to the best practices in intercultural development. Instructors should not only be aware of the theoretical frameworks of intercultural skills, but also understand how to support their students as they develop their skills during the GU program. It is also important for instructors to reflect on their own intercultural skills, especially when working with partners, and to set an example for their students to follow. Importantly, the training outcomes have seen instructors work closer with their students to raise intercultural awareness, as well as improving cooperation with partners.

The instructors have increased understanding of the intercultural skills needed for successful communication in the GU program. As a result, instructors can offer students more guidance and support in, for example, building peer rapport or coping with cultural differences. Furthermore, there is improved awareness of the transferable intercultural skills that are necessary when working with a variety of partners. Understanding the approach of a toolbox of skills, instructors are working to increase motivation by encouraging students to explore ways to overcome difficulties. As a result, there is a visible improvement among ECU students' communication with partners, both in and out of class.

There is also more recognition of setting an example and working closely with partners. Though the training was phrased in terms of improving student skills, instructors also have worked on improving their intercultural skills in the classroom. There is greater flexibility and understanding of the partners' situations. For example, ECU instructors have held classes earlier than scheduled to link with partners, and adopted innovative techniques to ensure classes continued throughout the COVID-19 lockdown for spring semester 2020.

\section{Conclusion}

There is a necessity for instructors in GVE to be given training in intercultural skills to deliver a successful program. Instructors, despite being experts in their specific fields and involved in the delivery of a GVE program for several years, benefited from work on intercultural skills. There is a need for structured training to introduce the fundamentals of intercultural skill development, as it allows the trainer not only to focus on their classroom methodology, but to also reflect on their own 
intercultural skills as required for the program. Importantly, such training has reinforced that soft skills are an integral part of the GU course outcomes and not simply by-products of student-student interaction.

As a result of the training described above, the GPE organization digitized the training materials into online workshops delivered through the Articulate 360 software. This has enabled the trainings to become interactive packages, which have been uploaded to a learning management system accessible to all partners in the GPE network. From the Fall semester of 2020, instructors participating in the GU program will be required to complete two trainings as part of the first of three badging levels - The Foundations of GU and How to Teach GU. These trainings cover the GU Core (the what), and give an overview of classroom methodology (the how). The How to Teach GU training covers such classroom activities as reflections, classroom management, and partner communication.

It is believed that structured training in intercultural skills for instructors of the GU program will work to benefit the quality, delivery, and outcomes of the program.

\section{References}

Ang, S., \& Dyne, L. V. (2008). Conceptualization of cultural intelligence: definition, distinctiveness and nomological network. In S. Ang \& L. V. Dyne (Eds), Handbook of cultural intelligence: theory, measurement and applications (pp. 3-15). M. E. Sharpe.

Ash, S. L., \& Clayton, P. H. (2009). Generating, deepening, and documenting learning: the power of critical reflection for applied learning. Journal of Applied Learning in Higher Education, 1(1), 25-48.

Bandura, E. (2007). Nauczyciel Jako Mediator Kulturowy. Tertium.

Byram, M. (1997). Teaching and assessing intercultural communicative competence. Multilingual Matters.

Byram, M., Kuhlmann, T., \& Muller-Jacquier, B. (2009, August 08). Intercultural competence assessment (INCA). European Website on Integration. https:/ec.europa.eu/migrant-integration/librarydoc/the-inca-project-intercultural-competenceassessment

COIL. (2019). Academic connections worldwide - COIL initiative. University of Washington Bothell. https://www.uwb. edu/globalinitiatives/academic/coil-initiative

Dyne, L. V., Ang, S., \& Koh, C. (2008). Development and validation of the CQS: the cultural intelligence scale. In S. Ang \& L. V. Dyne (Eds), Handbook of cultural intelligence: theory, measurement and applications (pp. 16-38). M. E. Sharpe. GPE. (2019). Global partners in education. https://thegpe.org/

Gudykunst, W., \& Kim, Y. (1992). Communicating with strangers. McGraw-Hill.

Kramsch, C. (1993). Context and culture in language teaching. Oxford University Press.

Ting-Toomey, S. (1999). Communicating across cultures. Guilford. 


\section{Virtual Exchange?}

Published by University of Groningen Press | UGP, a not-for-profit press

Groningen, The Netherlands | UGP@rug.nl

(C) 2020 UNICollaboration (collective work)

(C) 2020 by Authors (individual work)

Journal of Virtual Exchange 2020

Edited by Carolin Fuchs and Müge Satar

Special issue edited by Penelope Margaret Orton, Natesha L Smith-Isabell, and Mary Jane Radford Arrow

Publication date: 2020/12/11

Journal of Virtual Exchange (JVE) is an online, open-access, peer-reviewed journal aimed at practitioners and researchers in the field known variously as virtual exchange, telecollaboration, or online intercultural exchange. It is the official journal of UNICollaboration (https://www.UNICollaboration.org/), the international academic organisation dedicated to supporting and promoting telecollaboration and virtual exchange in higher-level education.

Rights. The whole volume is published under the Attribution-NonCommercial-NoDerivatives 4.0 International licence (CC BY-NCND 4.0); individual articles may have a different licence. Under the CC BY-NC-ND licence, the volume is freely available online for anybody to read, download, copy, and redistribute provided that the author(s), editorial team, and publisher are properly cited. Commercial use and derivative works are, however, not permitted.

Disclaimer. University of Groningen Press does not take any responsibility for the content of the pages written by the authors of this article. The authors have recognised that the work described was not published before, or that it was not under consideration for publication elsewhere. While the information in this article is believed to be true and accurate on the date of its going to press, neither UniCollaboration nor University of Groningen Press can accept any legal responsibility for any errors or omissions. Additionally, the publisher makes no warranty, expressed or implied, with respect to the material contained herein. While University of Groningen Press is committed to publishing works of integrity, the words are the authors' alone.

Trademark notice. Product or corporate names may be trademarks or registered trademarks, and are used only for identification and explanation without intent to infringe.

Copyrighted material. Every effort has been made by the editorial team to trace copyright holders and to obtain their permission for the use of copyrighted material in this article. In the event of errors or omissions, please notify the publisher of any corrections that will need to by incorporated in future editions of this article.

Typeset by Research-publishing.net (https://research-publishing.net)

Noto fonts are open source. All Noto fonts are published under the SIL Open Font License, Version 1.1. Noto is a trademark of Google Inc. (https://www.google.com/get/noto/).

ISSN: 2647-4832 (online only)

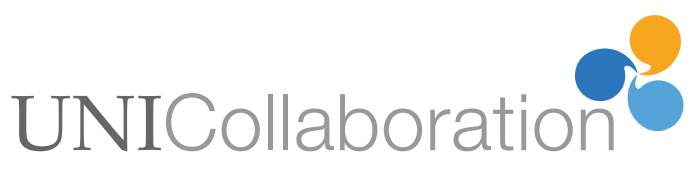

